\section{Pulmonary blastoma in an adult presenting as a chronic loculated effusion: a diagnostic problem}

\author{
Nyat-Kooi Chin, Chuen-Neng Lee, \\ Yoke-Sun Lee, Wan-Cheng Tan
}

\begin{abstract}
A 33 year old woman posed a diagnostic problem for two years with recurrent left sided chest pain and radiographic features suggestive of a loculated effusion. Diagnosis was finally made at thoracotomy which revealed an encapsulated cystic mass. Histological examination confirmed a rare monomorphic pulmonary blastoma.
\end{abstract}

(Thorax 1994;49:838-839)

Medicine

W-C Tan

Department of

Cardiothoracic

Surgery

C-N Lee

Department of

Pathology

Y-S Lee

National University Hospital, 5 Lower Kent Ridge Road, Singapore 0511

Reprint requests to: Dr W-C Tan.

Received 19 May 1993

Returned to authors

13 August 1993

Revised version received

25 October 1993

Accepted for publication

15 November 1993

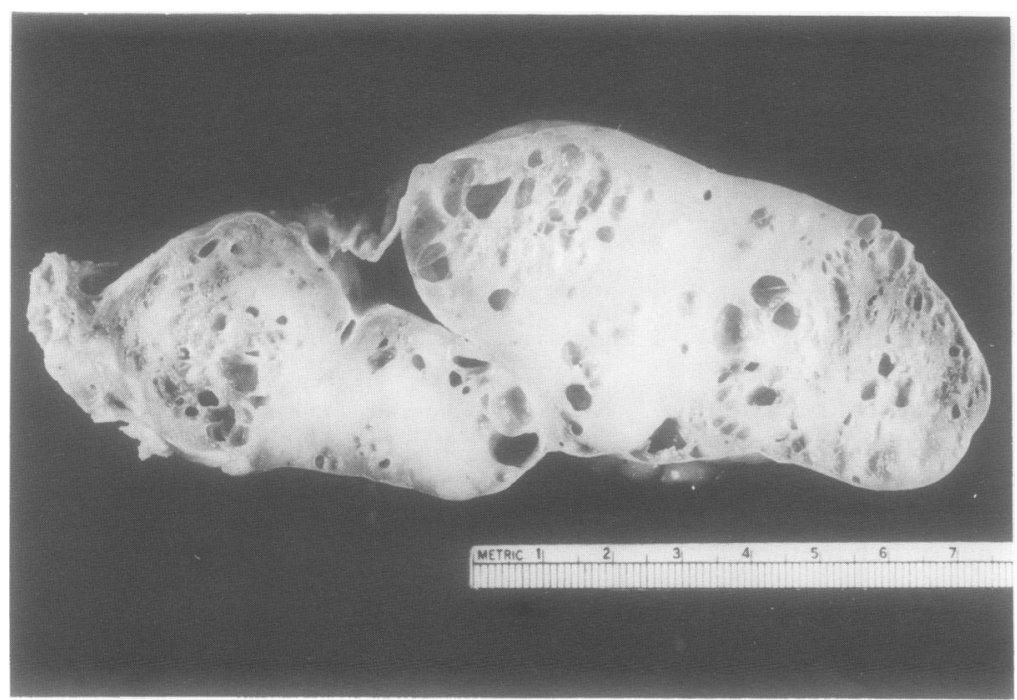

Figure 1 Resected specimen of oval shaped tumour mass with greyish solid and cystic areas.
Asia; secondly, she had survived two years without evidence of distant metastases; and thirdly, the histological examination showed a monomorphic pattern which is rare in the adult. ${ }^{3-7}$

\section{Case report}

A 33 year old Chinese woman presented in September 1990 with constant left sided pleuritic chest pain unrelieved by analgesics. There was no evidence of fever, cough, or weight loss. She was previously well and had never smoked. Her chest radiograph showed left basal consolidation with a loculated effusion, confirmed by computed tomographic scanning of the thorax. Pleural aspiration of $100 \mathrm{ml}$ bloodstained fluid was obtained after three attempts. This was a sterile transudate; cytological examination showed scanty neutrophils, lymphocytes, and benign mesothelial cells. The chest radiograph after aspiration showed little change. Mantoux testing with 10 units PPD was non-reactive. She was given a course of antibiotics with symptomatic improvement.

Six months later she developed a dry cough and low grade fever. Clinically she was well despite the persistent left pleural effusion. Peripheral blood counts were normal and the erythrocyte sedimentation rate was $10 \mathrm{~mm}$ in the first hour. Permission was not obtained for a repeat pleural aspiration. Fibreoptic bronchoscopic examination excluded endobronchial tumour and tuberculosis. She was nevertheless started on a course of antituberculous therapy comprising rifampicin, isoniazid, and ethambutol.

She completed nine months of treatment without complications but the chest radiograph remained unchanged. In November 1992 the dry cough, malaise, and weight loss recurred. She was dyspnoeic on moderate exertion and afebrile but physical examination revealed no new findings. A repeat thoracic computed tomographic scan showed a semisolid area in the posterobasal segments of the left lower lobe with an adjacent collection of fluid. The right lung and the mediastinum were normal.

At thoracotomy a large firm mass which occupied the left lower third of the pleural cavity and compressed the adjacent lung was found connected to the left upper lobe posteriorly by a pedicle. The remainder of the lungs, pleura, pericardium and mediastinum were normal. The gross specimen was oval in shape and weighed $424 \mathrm{~g}$. The cut section showed a greyish solid tumour with cysts varing from $1 \mathrm{~mm}$ to $20 \mathrm{~mm}$ in diameter (fig 1). Haematoxylin and eosin stained sections showed a lesion with solid and cystic areas. The solid areas were composed of loose spindle-shaped mesenchymal cells with cystic spaces and a varying degree of cellularity. Areas of increased cellularity appeared in sheets or as small nodules with scattered mitoses. Hyalinisation was seen in the stroma and particularly around blood vessels (fig 2). No epithelial, chondroid, or osteoid differentiation was identified. Immunohistochemically the tumour cells were vimentin positive, but negative for keratin, desmin, S-100, chromogranin, and NSE. The resection margin 


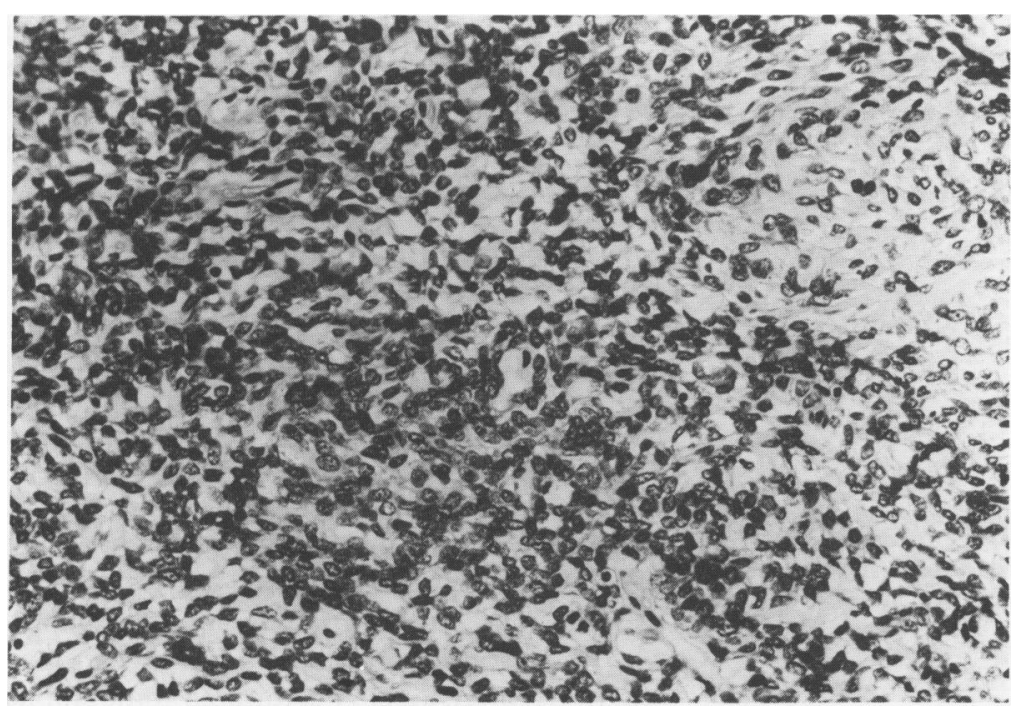

Figure 2 Section showing tumour composed of spindle-shaped mesenchymal cells with a focus of hyalinisation of the stroma. Stain: haemotoxylin and eosin. Magnification $\times 200$, reduced to $83 \%$ in origination. tremely poor prognosis. ${ }^{9}$ Histologically this case resembles childhood blastoma because of the lack of connective tissue differentiation. It is interesting that after two years the histological appearance has remained undifferentiated and the tumour has not metastasised. The behaviour of this apparently circumscribed tumour would suggest that it was relatively benign, despite the aggressive reputation of predominant stromal varieties.

The clinical presentation is non-specific with cough, chest pain, and dyspnoea. The usual investigations such as chest radiography, sputum cytology, bronchoscopic biopsy, or lymph node biopsy are of limited value. Tumour specimens obtained via thoracotomy or necropsy are usually necessary for a definitive diagnosis. The discrepancy between the radiological, clinical, and the intraoperative findings contributed to the diagnostic difficulty in this case. Clinically and radiologically the tumour occupied the lower third of the left pleural cavity, compressing the left lower lobe of the lung. At thoracotomy it was found to be attached by a long narrow pedicle to the left upper lobe from which it arose. The computed tomographic scan had failed to delineate this particular arrangement.

The best mode of treatment is not known, but surgical resection has so far been promising with postoperative survival varying from one month to 11 years. ${ }^{1-7}$ The role of postoperative adjuvant chemotherapy, irradiation, or both, is poorly defined, and the prognosis is uncertain as the number of cases reported so far has been small and methods of treatment varied. ${ }^{1-8}$

We are grateful to $\mathrm{Dr} \mathrm{R} \mathrm{M}$ du Bois for his comments and Professor B Corrin for reviewing the histology in this case. has been described in adults aged 15-77 years, with a slight male preponderance. ${ }^{1-7}$ Sporadic cases in younger patients have, however, been described. ${ }^{38}$

The histogenesis is still uncertain; it probably develops from a pleuripotential cell of one germ layer which later differentiates into mixed tissue components. The typical histological features are the gland-like structures lined by nonciliated epithelium, and the surrounding mesenchymal stroma is often interspersed with mixed malignant cells. The tumour may sometimes be encapsulated, often locally invasive, with regional and/or distant metastases. ${ }^{1-7}$ It can be differentiated from the pleuropulmonary blastoma of childhood in that the latter occurs in children under 15 years of age and usually undergoes sarcomatous change with an ex-
1 Barnard WG. Embryoma of lung. Thorax 1952;7:299. 1961;82:161-5.

3 Iverson RE, Straehley CJ. Pulmonary blastoma; long term survival of juvenile patient. Chest 1973;63:436-40.

4 Nazari A, Amir-Mokri E, Sarram A, Yaghmai I. Pulmonary blastoma. Chest 1971;60:187-9.

5 Bauermeister DE, Jennings ER, Beland AH, Judson HA Pulmonary blastoma, a form of carcinosarcoma: report of a case of 24 years' duration without treatment. $\mathrm{Am} \mathfrak{f}$ Clin case of 24 years' durati

6 Case records of the Massachusetts General Hospital: case 3 1984. N Engl f Med 1984;310:178-87.

7 Minken SL, Craver WL, Adams JT. Pulmonary blastoma. Arch Pathol 1968;86:442-6.

8 Ghaffar A, Vaidynathan SV, Elguezabal A, Levowitz BS Pulmonary blastoma: report of two cases. Chest 1975;67:600-2.

9 Manivel JC, Priest JR, Watterson J, Steiner M, Woods WG, Wick MR, et al. Pleuropulmonary blastoma: the so-called pulmonary blastoma of childhood. Cancer 1988;62:1516-26.
2 Spencer H. Pulmonary blastoma. I Pathol Bacteriol 